\title{
Hydrogen production technology by electrolysis of water and its application in renewable energy consumption
}

\author{
Jinyu $\mathrm{Li}^{1}$, Wanfu $\mathrm{Liu}^{1, *}$ and Wuqin $\mathrm{Qi}^{2}$ \\ ${ }^{1}$ School of Mechanical Engineering, Tianjin University of Commerce, TianJin 300134, China
}

\begin{abstract}
In order to deal with the energy crisis and environmental pollution, renewable energy power generation in the world has been rapid development. At present, the most widely used is solar energy and wind energy, but also caused a serious problem of abandoning light and wind. Hydrogen energy has become an ideal carrier of electric energy storage due to its high efficiency, clean and renewable characteristics. Electrolytic water hydrogen production technology with renewable energy as power source is one of the most promising energy conversion methods. This paper briefly analyzes the current situation of power generation and consumption of renewable energy in China in recent years, and then expounds the characteristics, principles, development status and improvement methods of alkaline, proton exchange membrane and high temperature solid oxide electrolytic water hydrogen production technology, and demonstrates its application prospect in the field of renewable energy power generation and energy storage with examples.
\end{abstract}

\section{Introduction}

Population growth and improved personal comfort are two important factors affecting the world economy. The increase in energy consumption and the production of more greenhouse gases (GHG) affect the fossil economy. In response to the energy crisis and environmental pollution, the solutions proposed around the world have focused on the production of renewable energy. At present, solar and wind energy are mostly used, but due to the volatility and intermittenency of their power generation and the unbalanced geographical distribution of load centres, the problem of wind and solar abandoning is serious. In order to match supply with demand and solve the problem of large-scale consumption of surplus power from renewable energy, it is essential to find a suitable way of energy storage. Conventional energy storage technologies include pumped energy storage, compressed air energy storage, battery energy storage and superconducting energy storage [1-2]. These conventional energy storage methods cannot meet the demand for super large capacity and long cycle consumption, but hydrogen storage has become the most potential energy storage technology currently due to its large capacity and transportability [3].

Hydrogen is hailed as the "ultimate energy" of the 21 st century due to its high efficiency, cleanliness and renewable advantages. At present, the main means of hydrogen production in China are still using fossil fuels, such as steam reforming, coal gasification and partial oxidation of heavy hydrocarbons. Electrolysis of water to produce hydrogen accounts for about $5 \%$, and other

\footnotetext{
* Corresponding author: 2540816932@qq.com
}

processes such as biological hydrogen production, photochemical hydrogen production, photobiological hydrogen production are still under development. Among them, only water electrolysis can be currently commercially available, because the equipment of water-electrolyser has modular characteristics, can realize the centralized production of hydrogen, and then photovoltaic, wind energy and other renewable energy are used as energy source to electrolysis water, not only green and renewable, but also can effectively solve the problem of super large scale energy consumption. In addition, the process of hydrogen production by electrolysis is simple, the product purity is high, and is conducive to the reduction and conversion of $\mathrm{CO} 2$, so it has a broad application prospect [4]. It is predicted that after $2050,70 \%$ of hydrogen will come from renewable energy, and electrolysis of water from renewable sources will become the mainstream.

\section{Current Situation of renewable Energy power generation and consumption}

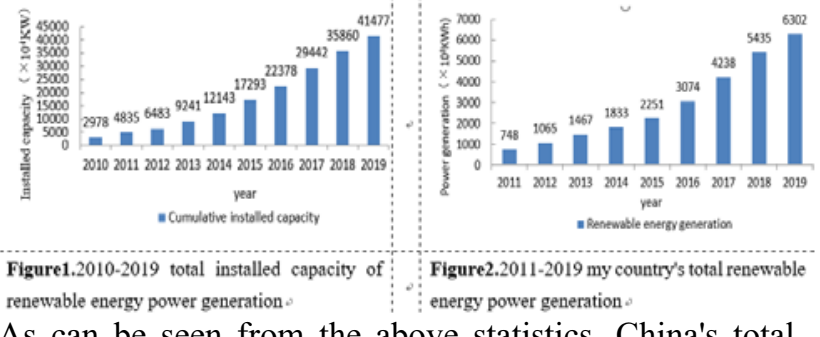

As can be seen from the above statistics, China's total installed capacity of renewable energy and power 
generation have been growing rapidly in recent years. By the end of 2019, the installed capacity has exceeded 400 million KW, with a year-on-year growth of $16 \%$, accounting for $20.6 \%$ of the country's total installed capacity. In 2019, the renewable energy generation capacity was 630.2 billion $\mathrm{KW} \cdot \mathrm{h}$ [5]. The development trends of the two is shown in Figure 1 and 2. The rapid development of total installed capacity and electricity generation has benefited from many new breakthroughs in renewable energy generation technology and equipment, but it has also caused grid connection pressure and large-scale consumption of abundant electricity. Only through centralized grid connection can no longer meet the current consumption demand, the curtailment of renewable energy in 2019 is still as high as 21.5 billion $\mathrm{KW} \cdot \mathrm{h}$, which is very serious. Besides, affected by the 2020 epidemic, the supply of renewable energy power generation equipment and project construction have been delayed to a certain extent, and the actual scale of grid connection is uncertain. On the other hand, electricity costs account for $80 \%$ of the entire cost of hydrogen production from electrolyzed water, which is the main factor restricting the promotion and application of hydrogen storage technology, now with the rapid expansion of the global renewable energy generation capacity, estimated after wind power, photovoltaic power generation cost will fall further, creating conditions for the large-scale application of hydrogen production technology from electrolysis of water. Taking the consumption as the starting point, when the renewable energy power generation system is a large-scale grid-connected, if there is still surplus power after it is supplied to the grid, the power can be distributed to the energy storage system through the control system, because the conventional energy storage method cannot meet the super large needs. Therefore, it is proposed to use centralized large-scale electrolysis equipment to achieve the elimination goal. The generated hydrogen can be used in chemical production, etc, or it can be connected to fuel cells to continue power supply. The process is shown in Figure 3(a); When the renewable energy power generation system is a small off-grid type, if there is still surplus power after it is supplied to independent users, in addition to the use of storage batteries, a distributed small electrolysis equipment can also be built to achieve consumption, as figure $3(\mathrm{~b})$.

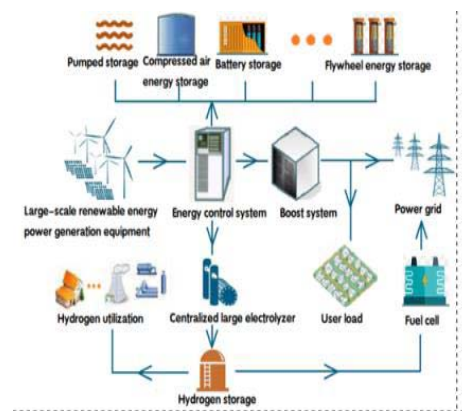

Figure 3(a). Consumption diagram of large-scale gridconnected renewable energy power generation

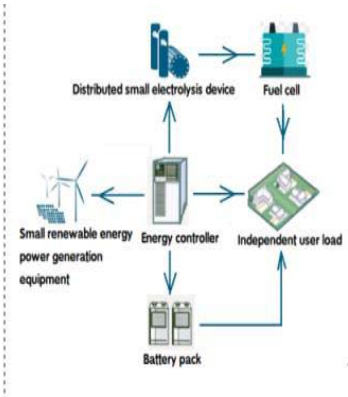

Figure 3(b). Consumption diagram of small offgrid renewable energy power generation

\section{Electrolytic water production technology \\ hydrogen}

Among the many hydrogen production technologies, electrolysis of water is still dominant today because it is the only technology that can directly utilize surplus electricity from renewable and fluctuating sources such as wind turbines or photovoltaic panels, which is the fundamental path to develop the hydrogen economy. Electrolytic water is a chemical process that is powered by electrical energy to decompose water into hydrogen and oxygen. The total reaction formula is:

$$
2 \mathrm{H}_{2} \mathrm{O}+\text { Electricity } \rightarrow 2 \mathrm{H}_{2}+\mathrm{O}_{2}
$$

The energy composition is as follows:

$$
\Delta H=\Delta G+T \Delta S
$$

Among them: $\triangle H$ is the enthalpy change, which is the total energy required for electrolysis of water. $\Delta \mathrm{G}$ is the change in Gibbs free energy, which reflects the minimum power supply required for the electrolysis

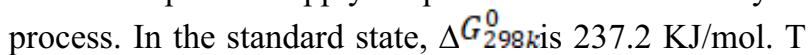
is the temperature of the reaction: $\Delta \mathrm{S}$ is the entropy change of the reaction; $\mathrm{T} \Delta \mathrm{S}$ reflects the heat energy absorbed by the electrolysis process.According to the relationship between reversible battery electromotive force $\mathrm{E}$ and Gibbs free energy $\Delta \mathrm{G}$ :

$$
\Delta G=-n E F
$$

Among them:n is the number of electron transferred; $F$ is Faraday constant.Thus, in the standard state, the theoretical decomposition voltage of water is $1.23 \mathrm{~V}$ and the corresponding minimum power consumption is $2.95 \mathrm{kwh} / \mathrm{m} 3$.For reaction (1), the electrolytic efficiency is defined as follows :

$$
\eta=\frac{W_{\mathrm{t}}}{W_{\mathrm{s}}}
$$

Among them,Wt represents the theoretical power consumption for generating hydrogen per standard square, that is $2.95 \mathrm{KWh}$; Wa represents the actual power consumption for generating H2 per standard square. In the actual electrolysis system, due to the influence of electrode materials, diaphragm, electrolyte and other factors, there will be a certain ohmic loss, resulting in the actual power consumption is far greater than the theoretical power consumption, and the electrolysis efficiency is less than 1. At present, there are three main electrolytic water technologies: alkaline electrolysis, proton exchange membrane (PEM) electrolysis, and high temperature solid oxide electrolysis (SOE). For alkaline and PEM electrolysis, the energy consumed in the electrolysis process is provided by electrical energy, while SOE has a higher operating temperature, which requires not only electrical energy, but also hightemperature heat energy.

\subsection{Alkaline electrolytic}

Alkaline electrolysis is the most mature electrolytic water hydrogen production technology, and has been 
widely used. The electrodes in the alkaline electrolysis cell are generally metal alloys, such as RaneyNickel and Ni-Mo alloy, etc, to decompose water. The electrodes are immersed into an alkaline solution (usually $\mathrm{KOH}$ ) with a concentration of $20 \% \sim 30 \%$, and the diaphragm is used to separate the gas. The operating temperature of the alkaline electrolysis cell is usually controlled at $70 \sim$ $80^{\circ} \mathrm{C}$, and the pressure is $0.1 \sim 3 \mathrm{MPa}$ [6]. The current electrolytic cell structure mainly has two types: unipolar and bipolar.

The advantages of alkaline electrolysis technology are that the process is simple, easy to operate, does not need expensive catalysts, and has a long life. Its disadvantages are low efficiency, high ohmic loss in liquid electrolyte and low current density, so this method consumes a large amount of electric energy, Each bidder' s hydrogen production consumes about $4.5 \sim 5.5 \mathrm{KW} \bullet \mathrm{h}$. Resistance mainly comes from external circuit resistance, transmission resistance and electrochemical resistance, among which transmission resistance is the main cause of high ohmic loss, such as the bubbles generated on the electrode surface and the influence of diaphragm on electron conduction [7]. At present, the main concern is the development of alkaline electrolysis cells. In order to improve the efficiency, it is possible to accelerate the escape of bubbles by using electrolyte circulation, making porous electrodes or adding inert surfactants; In addition, a new diaphragm materials-inorganic membrane and the new electrolytic cell structure - zero spacing structure can be developed to reduce ohmic loss [8].

\subsection{Proton exchange membrane electrolysis}

The key of proton exchange membrane (PEM) electrolysis technology lies in proton exchange membrane, which is an organic film with strong chemical stability, proton conductivity and good gas separation. The membrane electrode assembly (MEA) is formed by the diaphragm and porous electrode composed of catalysts on both sides of the distribution. The working principle of PEM electrolytic cell is that Water is oxidized into $\mathrm{O} 2$ at the anode and $\mathrm{H}+$ is released. $\mathrm{H}+$ passes through the proton exchange membrane in the form of hydrated protons and is reduced to hydrogen at the cathode.

PEM electrolysis has the advantages of safety, cleanliness and high efficiency. Compared with alkaline electrolysis, it has low ohmic loss, high current density and hydrogen purity, and higher flexibility and reactivity in the operation of the device, so the power consumption is reduced to some degree. Each bidder's hydrogen generation consumes about $3.8-5 \mathrm{KW} \cdot \mathrm{h}$. The disadvantages are high cost and short life, so it is currently only suitable for small-scale hydrogen production. The reason is that most of the proton exchange membranes currently used are fully sulfonic acid type membranes, which are highly acidic when infiltrated by water. Moreover, due to the higher theoretical oxygen evolution potential, most metals will corrode at this potential, so Pt noble metals are mostly used as catalysts for the anode and cathode. In addition, the cost of proton exchange membrane is also relatively high, and such exchange membrane is prone to degradation during use, so the membrane life is short [9]. The high cost of these materials hinders the industrial application of PEM electrolysis technology. At present, the main problem is to find more durable proton exchange membrane materials in thermodynamics and chemistry, and reduce the diffusion coefficient of membrane solid phase. For noble metal catalysts, the cost can be reduced by reducing catalyst load and developing alloy catalyst.

\subsection{High temperature solid oxide electrolysis}

According to the working temperature of the electrolysis device, the above two electrolysis methods belong to the low temperature type, and the working temperature is about $80^{\circ} \mathrm{C}$. The hydrogen production efficiency can reach $60 \%-75 \%$ and $70 \%-85 \%$ respectively. The main problem is high power consumption. However, the working temperature of high temperature solid oxide electrolysis (SOE) is $800-950^{\circ} \mathrm{C}[10]$. Because it works at high temperature, heat energy can replace part of the electric energy, and the power consumption is further reduced. Each bidder's hydrogen generation consumes about $2.6-3.6 \mathrm{KW} \cdot \mathrm{h}$, so the electrolysis efficiency is very high, up to more than $90 \%$. The structure of SOE is developed from tubular structure to flat plate structure, with higher energy density and no need for noble metal catalyst, so the cost is not high. The working principle is that water enters the electrolytic cell in the form of steam and generates $\mathrm{H} 2$ and $\mathrm{O} 2-$ on the cathode side. O2reaches the anode through the electrolyte layer and generates $\mathrm{O} 2$. The function of electrolyte is to allow oxygen ions to permeate but prevent gas from permeating.

Since more than two-thirds of the cost of electrolysis water comes from the use of electricity, operating at higher temperatures can reduce the cost of producing hydrogen, especially if some of the electricity needed can be replaced by external heat sources (such as nuclear energy, renewable energy or waste heat from hightemperature industrial processes). Higher temperature also improves electrode activity, and helps to reduce electrode overpotential, increase current density, and reduce power loss, all of which reflect the good thermodynamic and kinetic performance of SOE at high temperature. However, due to the high operating temperature, the oxygen electrode of conventional materials will undergo anodic polarization and delamination, resulting in high voltage loss of the oxygen electrode, poor stability of the electrolytic cell material, reduced life and other problems, which are still in the research stage. If breakthroughs are made in new materials and new oxygen electrodes, SOE is expected to be an important way to produce hydrogen efficiently. 


\section{Application of Hydrogen Storage Technology by Electrolysis of Water}

In terms of hydrogen energy storage technology, Europe is relatively mature in development, with complete technical reserves and equipment manufacturing capacities, as well as a number of demonstration projects for hydrogen energy storage systems to be used in conjunction with new energy access. A power company in California has built a solar-cell hydrogen refuelling station that uses solar power to electrolyze water to produce hydrogen. In Japan's WE-NET program, the PEM electrolyser has been able to work continuously for more than 4000 hours at an efficiency of $90 \%$ under the working conditions of $80^{\circ} \mathrm{C}$ and $1 \mathrm{~A} / \mathrm{cm} 2$. France started and completed the MYRTE project in Corsica, which combines photovoltaic power generation and hydrogen storage, so as to average the power output of photovoltaic power stations and make it easier to integrate into the grid.

From the domestic situation, China has just launched relevant construction in the demonstration application of hydrogen energy storage systems, mainly for demonstration of new-energy vehicles and distributed power supply. At present, there are four $35 \mathrm{MPa}$ hydrogenation stations in China, and more large-scale hydrogenation stations are under construction. There are three main wind power coupling projects at this stage. One is the "Technical Research and Demonstration of Direct Hydrogen Production from Wind Power and Fuel Cell Power Generation System" project of China Energy Conservation and Environmental Protection Corporation. The hydrogen production power of this project is $100 \mathrm{~kW}$, and the fuel cell power generation is $30 \mathrm{~kW}$. Second, the largest wind power hydrogen production demonstration project in China, which is cooperated by China and Germany, is to build a 10MW electrolytic water hydrogen production system in Guyuan, Hebei, and to cooperate with a $200 \mathrm{MW}$ wind power plant to produce hydrogen. The third is the project of $100 \mathrm{MW}$ wind power installed capacity and 10MW hydrogen energy storage capacity approved by Gold-wind Technology in Jilin. The State Grid Corporation of China has also carried out relevant projects and studies. Among them, the "Preliminary Study of wind-solar combined with seawater hydrogen Production Technology" project has proposed a variety of application schemes for wind power and photoelectric hydrogen production. Such as Donghai Wind Farm, the comprehensive benefit evaluation of wind and photoelectric hydrogen production has been carried out. The Smart Grid Research Institute of State Grid has also launched the "research on key technologies of hydrogen storage and its application in new energy Access", preliminarily possessing the test capability of hydrogen energy storage system.

\section{Conclusion}

Electrolyzed water technology is a technology that can directly utilize surplus power from renewable energy sources such as wind turbines or photovoltaic panels and other fluctuating energy sources. It has become a strategic reserve technology that domestic and foreign large energy companies focus on developing and gradually realize large-scale application. Among them, hydrogen production by alkaline electrolyzed water is currently the most mature hydrogen production method, but it has problems such as pollution and low efficiency. Studying new electrode and diaphragm materials is an important way to improve efficiency, which can be used to stabilize power grid or directly connect to large-scale wind energy or solar energy farms. For PEM electrolyzers with better dynamics and gas mass, the development of new non-precious metal catalysts and new proton exchange membrane technologies is the key, which can be used as intermediate energy buffers at the industrial plant or residential level; Now, high temperature solid oxide electrolysis is in the early stage of development. If it can solve the problem of limited electrolytic cell materials caused by high temperature operation, it will become the most competitive hydrogen production method from electrolysis of water later.

Whatever the technology, the research goal should be to improve efficiency and reduce costs. These include the study of electrode materials, electrocatalysts and diaphragms. However, the current commercial penetration rate of water electrolysis is not high, which is mainly due to its high power consumption and related high operating costs. In order to realize its large-scale application, a series of researches in the production, storage, transportation should be carried out.

\section{References}

1. J.P. Barton, D.G. Infield.,Energy storage and its use with intermittent renewable energy[J]. IEEE Transactions on Energy Conversion, (2004), 19(2): 441-448.

2. W.L.Zhang, M. Qiu, X.K. Lai, Application of energy storage technology in power system $[\mathrm{J}]$.Power System Technology, (2008), 32(7): 1-9.

3. S.J. Mou, J. Lin, X.T. Xing, High-temperature solid oxide electrolysis of water to produce hydrogen and energy storage technology and its application prospects [J]. PST, (2017), 41(10): 3385-3391.

4. Liu Yun. The progress of green energy hydrogen energy and hydrogen production by electrolysis of water [J]. Power Tech, 2012, 36(10): 1579-1581

5. Polaris Energy Storage Network. China New Energy PowerGenerationAnalysisReport.(2020)

6. R.F.Souza,J.C.Padilha, Electrochemical hydrogen production from water electrolysis using ionic liquid as electrolytes: Towards the best device[J]. J. Power Sources, (2007), 164: 792-798

7. A. Roy, S. Watson, Comparison of electrical energy efficiency of atmospheric and high-pressure electrolysers[J]. Int. J. H E, (2006), 31:1964-1979

8. Y.H.Li, J.Chen,.Research progress in hydrogen preparation technology[J]. E.F, (2019), 41: 22-27 
9. M.K.Debe, Initial performance and durability of ultra-low loaded NSTF electrodes for PEM electrolyzers [J]. JTES, (2012), 159(6): 165-176.

10. M.A. Laguna, Recent advances in high temperature electrolysis using solid oxide fuel cells: a review $[\mathrm{J}]$. Journal of Power Sources, (2012), 203(203): 4-16 